\title{
TV/Series
}

$7 \mid 2015$

Le Pilote et la chute

\section{The X-Files (1993-2002) ou le jeu des niveaux narratifs}

Frédéric Gai

\section{(2) OpenEdition \\ 12 Journals}

Édition électronique

URL : http://journals.openedition.org/tvseries/280

DOI : $10.4000 /$ tvseries. 280

ISSN : 2266-0909

Éditeur

GRIC - Groupe de recherche Identités et Cultures

Référence électronique

Frédéric Gai, «The X-Files (1993-2002) ou le jeu des niveaux narratifs », TV/Series [En ligne], 7 | 2015, mis en ligne le 01 juin 2015, consulté le 01 mai 2019. URL : http://journals.openedition.org/ tvseries/280 ; DOI : 10.4000/tvseries.280

\section{(c) (1) $(9)$}

TV/Series est mis à disposition selon les termes de la licence Creative Commons Attribution - Pas d'Utilisation Commerciale - Pas de Modification 4.0 International. 


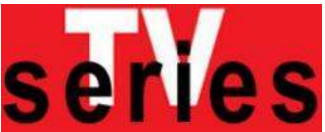

The $X$-Files (1993-2002) ou le jeu des niveaux narratifs Frédéric GAI

The X-Files ne cesse de questionner les limites du début et de la fin : les épisodes fermés sont une suite d'enquêtes policières qui, bien qu'arrêtées, restent bien souvent en suspens, donnant un peu plus de valeur et de pérennité au service des affaires non classées; les épisodes de la mythologie élargissent le champ des interrogations, passant d'un complot fomenté autour du secret des extraterrestres au sort de l'humanité, aussi bien sa naissance, que sa fin annoncée. La série a donc su explorer différentes voies narratives, proposant durant neuf saisons boucles et relances, et usant du cliffhanger en fin de saison, repoussant ainsi l'achèvement de sa diffusion, ou en faisant du premier long-métrage diffusé en salles aussi bien un blockbuster traditionnel qu'un « épisode » décroché du cycle de diffusion de la Fox.

The X-Files keeps questioning the limits of beginning and ending: the closed episodes are a sequence of police investigations and, although they do have an ending, they often remain unresolved however, and give a little more value and sustainability to the department of unsolved cases. Moreover, the mythology episodes extend the range of questions, switching from a conspiracy fomented around the secret about the aliens to the fate of humanity, including both its birth and extinction. The series has thus been then able to explore different narrative paths. It provided loops and reminders during nine seasons, sometimes used cliffhangers and thus postponed its own completion. It likewise turned the first feature film distributed in cinemas into an " episode » dropped out of the Fox release cycle.

$T$

he $X$-Files est une série qui n'a eu de cesse de repousser sa fin. Débutée en 1993 dans un relatif anonymat, elle a surfé sur un succès rapide qui a connu son apogée au cœur des saisons 4,5 et 6 , créant un engouement rare dans le monde de la télévision. Rien qu'à ce titre, la création de Chris Carter, entouré d'une équipe de scénaristes particulièrement actifs et brillants et de deux figures - David Duchovny et Gillian Anderson - qui ont construit leur carrière sur les rôles de Mulder et Scully, peut interroger le thème du pilote et de la chute. Sans pour autant aborder de front les conflits qui ont opposé l'équipe de réalisation, la production et le diffuseur, en l'occurrence la puissante network qu'est la Fox, ce rapport n'a eu de cesse d'interroger les modes de conclusion, pour ne pas dire le principe de dénouement ${ }^{1}$ d'une série qui, dans sa construction même, manipule les notions de début et de fin, les étire et les retourne à outrance. C'est justement cette double perspective, d'une part narrative et discursive, et d'autre

\footnotetext{
${ }^{1}$ Le dénouement n'est pas une fin en soi et il ne cesse de (dé)mêler but et fin, « deuil », « héritage» et « avenir ». Sur ce sujet, voir Lionel Ruffel, Le Dénouement, Lagrasse, Verdier, « Chaoïd », 2005, p. 28 et 34.
} 
part ce qui pourrait appartenir aux contingences de la diffusion, aux conséquences du succès sur les modes et les raisons de continuation de la série, qui retiendra notre attention. Si la première des deux prendra largement le pas sur la seconde, notamment dans l'idée de mettre en avant le « jeu des niveaux narratifs », notre volonté est avant tout de ne pas omettre ce qui appartient en propre à l'industrie de la télévision, dans l'optique de montrer en quoi l'écriture d'une série telle qu' $X$-Files relève d'une dynamique générale et d'une intentionnalité qui, paradoxalement, n'est jamais totalement arrêtée. En somme, les questions auxquelles nous tenterons d'apporter une réponse pourraient être formulées de la manière suivante: quelles sont les limites d'une série telle qu'X-Files ? Comment son succès et sa dimension "mythologique» ont permis, voire appelé, des déclinaisons ? Comment les problématiques structurelles de début et de fin ont-elles été intégrées à l'intérieur même de la série, au point de devenir un axe de réflexion poétique et métaphysique? Car il est essentiel de ne pas oublier un élément lorsqu'on évoque $X$-Files : repousser la fin n'est pas uniquement un problème de production et/ou de diffusion, mais bien une donnée générique de la série, un axiome développé tout au long de sa création. Rappelons-nous en effet que la tagline ("The truth is out there »), qui conclut chaque fin de générique ou presque (et donne le ton de l'épisode), incite à revoir nos certitudes spatiales et temporelles : en somme à repousser les limites, à visiter les frontières d'un réel dans tous ses états. Â mener une quête moderne où toutes les échelles du monde sont intégrées.

\section{La dualité structurelle d'X-Files : entre série policière et fiction mythologique}

Sans trop dévoyer ce qui constituera notre propos central, la pérennité d'X-Files, au-delà des sujets empruntés à la science-fiction et aux enquêtes policières, mais aussi des angoisses d'une époque qu'elle fait résonner dans chaque téléspectateur ${ }^{2}$, sa longévité tient en la structuration générale de sa narration et en la capacité des créateurs à faire rapidement jouer les différents niveaux offerts par le format de la série : l'épisode, voire les groupes d'épisodes, la saison, la série dans son intégralité. Dans son ensemble, $X$-Files suit en effet deux modèles qui se déploient conjointement et, parfois (mais parfois seulement), peuvent s'imbriquer.

\footnotetext{
${ }^{2}$ Frédéric Gai, "The $X$-Files : allégorie de la condition postmoderne du monde ", in TV/Series, $\mathrm{n}^{\circ} 1$, http://www.univ-lehavre. fr/ulh_services/Numero-1Issue-1.html.
} 


\subsection{La construction progressive de l'arc mythologique}

Le téléspectateur suit une sorte de feuilleton marqué par la quête des deux personnages principaux des preuves de l'existence des extraterrestres. Fortement inspirés par les thèmes de la théorie du complot et par un profond pessimisme envers l'administration hérité des années post-Watergate, ces «lots» d'épisodes fonctionnent comme une suite de longs-métrages situés à des points stratégiques du déroulement de la saison afin de construire et de développer l'arc mythologique de la série. Outre les doubles épisodes, qui ponctuent et animent chaque saison et créent un effet de suspens d'une semaine sur l'autre, les créateurs ont aussi institué le principe du cliffhanger, c'està-dire d'un épisode se terminant au cœur l'action, imposant au téléspectateur une forte tension, qui peut perdurer jusqu'au début de la saison suivante. Le long-métrage vient ainsi cheviller les saisons entre elles. Ce modèle narratif donne d'abord l'impression que les deux agents du FBI progressent dans une enquête au long cours. À cette sensation «feuilletonnante » première vient s'adjoindre l'idée d'une complexité qu'il faut expérimenter, les sujets abordés (les extraterrestres, le complot gouvernemental, le sort de l'humanité) étant des problématiques aux multiples facettes qui ne sauraient être ramenées du côté de l'univocité. Le téléspectateur avance, mais prend aussi la mesure de ce qu'on lui raconte. En effet, si les «preuves » s'accumulent, l'échelle du complot et de son implication sur le sort du monde ne cessent, eux, de grandir. L'enquête policière sur l'existence des «petits hommes verts» devient alors une tragique chasse au papillon afin de répondre à l'équation du monde...

\subsection{Les contes policiers fantastiques}

Le second modèle adopté est d'abord inspiré de la série policière à proprement parler. Le téléspectateur suit chaque semaine le couple d'agents du FBI qui, selon une tradition de genre, est plongé dans une enquête spécifique, avec un thème et une image bien définis. La série fait de réguliers clins d'œil au freak show, autrement dit au monstre de la semaine. L'un des épisodes les plus représentatifs, mais aussi l'un des plus réussis, de cette veine d'épisodes est très certainement The Post-Modern Prometheus (5.5, fig.1). Certes, tous les épisodes ne reprennent pas le ton divertissant qui a marqué le freak show américain jusqu'à la première partie du $\mathrm{XX}^{\mathrm{e}}$ siècle, et la série se veut parfois plus sombre en décloisonnant les codes du genre et en faisant intervenir des thèmes modernes empruntés à la science-fiction. Reste que le contexte géographique des épisodes (l'Amérique profonde pour le dire rapidement), qui se pare quelquefois de ses costumes de carnaval, ainsi que le thème de l'anomalie, qu'elle relève d'une nature 
"déviante » ou d'une manipulation scientifique, marquent clairement l'angle de vue de cette deuxième partie de la série. Ces épisodes peuvent aussi constituer de véritables variations sur le cinéma de genre, des histoires de vampires aux esprits malveillants en passant par les dérives technologiques. La série se fait alors panel d'histoires détachées, accentuant ainsi la sensation que nous suivons le quotidien peu commun de deux agents du FBI confrontés aux errements du monde. Entre les épisodes consacrés à la mythologie extraterrestre, Mulder et Scully parcourent les États-Unis en long, en large et en travers, tentent de confondre sectes et autres faux prophètes, combattent des machines intelligentes ou une nature devenue hostile, à chaque fois en mettant leurs convictions spirituelles et/ou scientifiques à l'épreuve. D'un point de vue structurel, le téléspectateur est confronté à des épisodes fermés, dont le sujet n'est pas développé ailleurs. Bien sûr, ce choix d'une suite de " contes policiers fantastiques » détachés n'interdit pas à la narration d'opter pour des fins ouvertes ou en suspens, faisant ainsi écho à la tradition du récit de science-fiction.

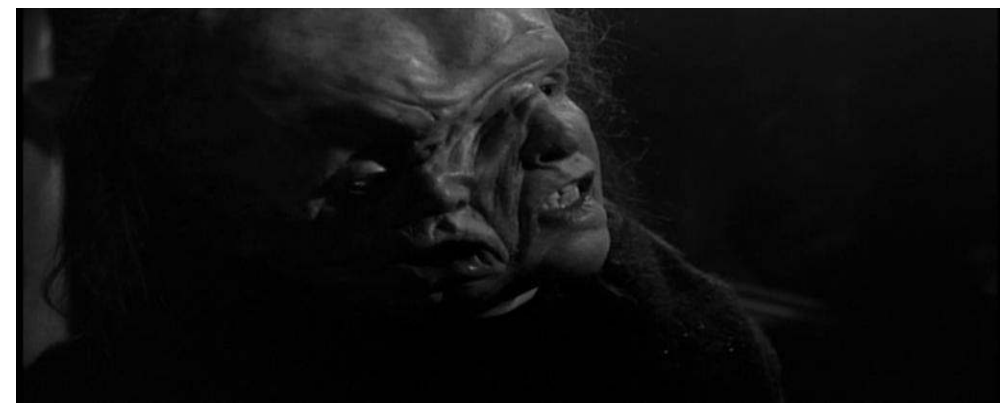

Figure 1 : le « Grand Mutato » interprété par Chris Owens

1.3. Une saison de gestation

Il est clair que le feuilleton mythologique prend largement le pas sur la multitude d'épisodes fermés, comme le prouve l'image laissée à la postérité par la production. Par ailleurs, le sujet est prégnant dès le pilote, où les deux agents sont amenés à Bellefleur, dans l'Oregon, pour enquêter sur une série d'enlèvements touchant une promotion du lycée de la ville. Tout au long de la première saison, la série tend même à traiter toutes les facettes de la thématique extraterrestre, des enlèvements à la manipulation génétique, en passant par les batailles technologiques. Néanmoins, si l'importance du sujet n'est pas contestable dès les premiers instants de la diffusion, l'alternance des épisodes est loin d'être visible dans la première saison qui tend même à être bouclée. Certains épisodes mêlent alors allègrement les deux pans de la série, comme Masculin/Féminin (1.14) ou Ève (1.13), au cours 
desquels le caractère paranormal alterne entre anomalie monstrueuse " terrestre » voire " folklorique » et théorisation autour du mythe des extraterrestres et de la manipulation génétique. Finalement, à ce stade de sa création, nous n'en sommes qu'à un état matriciel de la série : tout est encore en gestation dans les épisodes et il faut pouvoir trouver les voies de développement et de pérennisation du sujet. À l'inverse, la fin de la sixième et le début de la septième saison viennent ramener la pluralité des intrigues de la série dans le giron de sa propre mythologie. En effet, les découvertes faites par Scully en Côte-d'Ivoire accréditeraient l'idée que tout phénomène, habituellement traité sous un angle mystique, ésotérique, spirituel, religieux, technologique, monstrueux, etc., serait extraterrestre. La spécificité de la première saison est enfin sa structure générale bouclée, structure qui détonne avec le reste de la production. En effet, le premier et le dernier épisode se terminent sur le même plan-séquence au cours duquel le téléspectateur suit l'énigmatique Homme à la cigarette ranger des preuves dans un hangar du Pentagone (fig.2), installant par là une sensation d'enfermement et l'idée d'une impossible quête, et surtout ne faisant pas d'un double épisode « mythologique » la cheville entre deux saisons. 


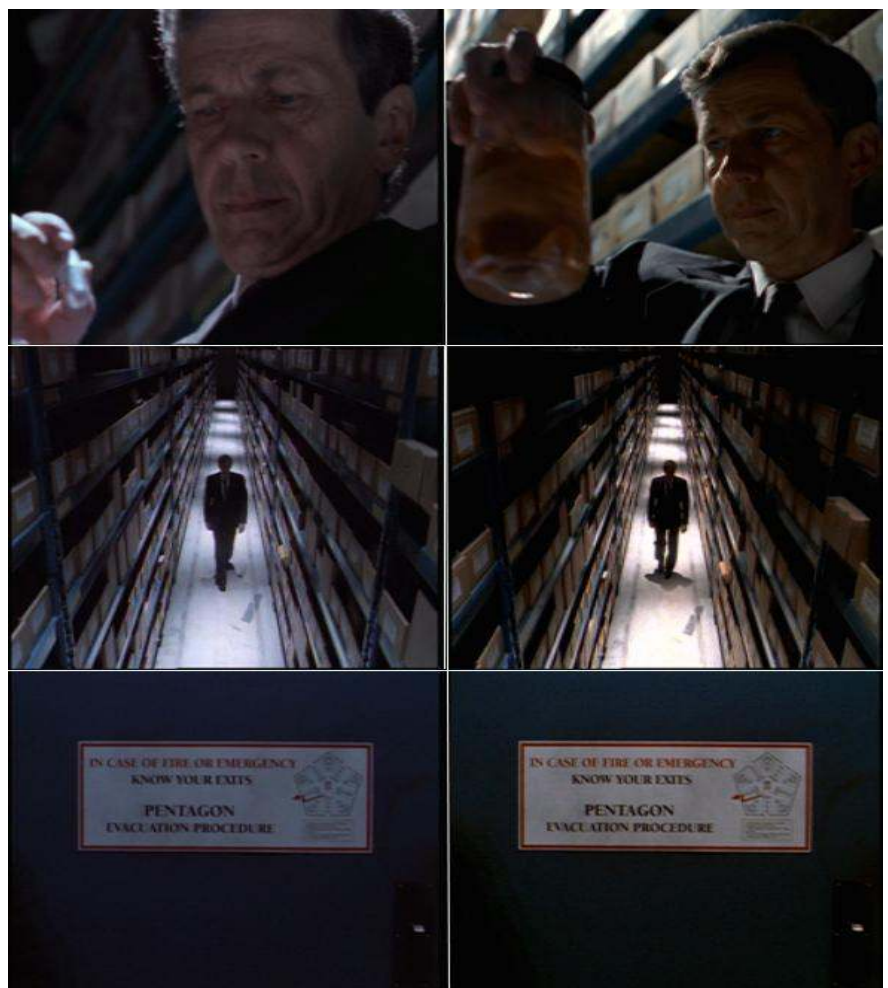

Figure 2 : fins des premier et dernier épisodes de la première saison

Le constat effectué sur cette première saison tend à contester partiellement l'idée d'une intentionnalité de la mythologie qui est finalement permise par le succès progressif de la série et par la possibilité de sortir en VHS les premiers longs-métrages, et que la deuxième saison va commencer à développer, avec Duane Berry 3 (2.5) et Ascension (2.6), puis Colony (2.16) et End game (2.17). Il est même bon de remarquer que le premier double épisode de la série est consacré à Eugène Tooms, monstre génétique qui se régénère en mangeant le foie de ses victimes (1.3 et 1.21, fig.3). La reprise du personnage est d'ailleurs reconstituée a posteriori en long-métrage lors de la sortie VHS, les deux épisodes étant séparés dans la chronologie de la narration et de la diffusion. C'est donc un

3 Une contingence majeure pousse aussi Chris Carter à provoquer l'enlèvement de Scully et le retrait temporaire de Gillian Anderson du casting : la grossesse de l'actrice. 
«monstre » et non un «extraterrestre » qui connaît le premier les honneurs de ce que nous pourrions en toute logique appeler un produit dérivé.

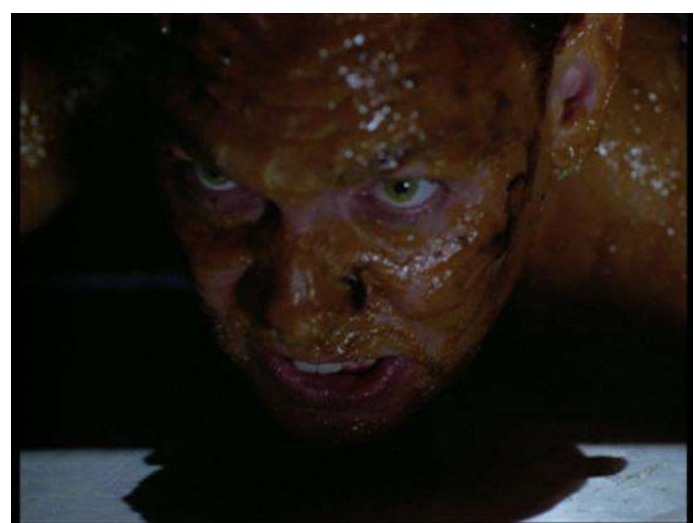

Figure 3 : Eugène Tooms (Doug Hutchison), premier monstre d' $X$-Files

\subsection{Le succès et ses dérives}

Puisqu'il est question de dérivation, tant dans son acception poétique que marketing, nous nous permettrons une rapide exploration en dehors même de la série. Une des particularités et des preuves du succès d' $X$-Files est la réalisation de films qui tous deux illustrent parfaitement les deux pans de la série et en questionnent les modes de continuation. Le premier, Fight the future, sorti dans les salles en 1998, s'inscrit résolument dans la mythologie de la série, tout en se voulant un véritable blockbuster hollywoodien, ce qui réoriente sensiblement l'esthétique de la série, d'abord en déplaçant le lieu de production de Vancouver à Los Angeles. Par ailleurs, le film s'installe entre deux saisons, la cinquième, qui s'achève sur un épisode intitulé The End (5.20), et la sixième, commencée par The Beginning (6.1). En somme, si la diffusion change, le modèle narratif ne déroge pas pour autant à ses habitudes, les créateurs prenant même leurs distances avec d'autres productions de ce type telles qu'Independance Day ou Men in Black ${ }^{4}$. Finalement, Chris Carter tente le pari d'intégrer son

4 Dans la première partie de Fight the Future, Mulder, dépité par son destin peu enviable d'enquêteur incompris, se saoule dans un pub de Washington, avant d'uriner sur l'affiche du film de Roland Emmerich. Dans The Beginning, les deux agents voient leur affaire comparée au scénario de Men in Black, film que Mulder avoue n'avoir jamais vu. 
film à la série, tout en proposant aux néophytes un divertissement somme toute conventionnel.

Le second film, I Want to Believe, sorti quant à lui en 2008, ramène l'univers d' $X$-Files du côté du film de genre, marqué par une enquête policière sur fond de croyance religieuse et de manipulation génétique. Situé dans la continuité de la série, il permet toutefois de poursuivre l'exploration des deux personnages, ce qui constitue une grande part du propos des saisons 8 et 9, et de remarquer que leurs obsessions spirituelles et métaphysiques sont pérennes et qu'elles sont loin d'être épuisées. Enfin, le film opère un retour esthétique aux sources, notamment en jouant sur les décors extérieurs et sur une photographie en clair-obscur. En mettant ces deux productions en miroir, les créateurs exposent leur conscience de leur propre création, de son esthétique et des possibilités qu'elle offre. Ils continuent, en somme, d'explorer les possibilités offertes par les différents niveaux narratifs de la série.

Le troisième volet, annoncé depuis quelques années déjà, mais officiellement bloqué pour des problèmes de financement, pose à sa façon les questions du début et de la fin. Alors qu'une orientation «mythologique» semble avoir été prise dans les ébauches de scénarios, les créateurs tendent apparemment à décliner ce qui est devenu au fil des années une franchise qu'ils veulent conclure, autant que faire se peut. Toutefois, en enchaînant les effets d'annonce, soit par l'intermédiaire des créateurs eux-mêmes ou par l'entremise des deux acteurs principaux, $X$-Files entretient son propre mythe auprès de sa communauté de fans, encore très active à ce jour. Cette pratique fait de l'univers de la série un produit commercial encore rentable, mais surtout un véritable phénomène de société. L'annonce de la diffusion d'une dixième saison pour début 2016, qui s'inscrit auprès de Twin Peaks ou de 24 heures dans un mouvement de reprise d'anciens programmes, insiste justement sur les capacités esthétiques et commerciales, ainsi que l'inscription dans la durée de la série.

Enfin, permettons-nous un dernier détour qui peut mettre en avant la problématique du succès pour la continuation et les déclinaisons d'une production. Face à la réussite, mais aussi à la fin programmée d'X-Files, Chris Carter décide de lancer en 2001 un spinoff, The Lone Gunmen (2001). Une saison et treize petits épisodes plus tard, la production diffusée par la Fox est arrêtée. Ainsi, la destinée des trois acolytes paranoïaques, commencée dans $X$-Files, continuée dans une autre série, se termine-t-elle... dans $X$-Files, leur mort survenant dans la dernière partie de la neuvième saison, l'épisode Jump the Shark (9.15) fonctionnant alors comme un cross-over5. Si X-Files n'a

5 Chris Carter est un adepte du genre puisqu'il fait apparaitre Franck Black, le personnage principal de sa série Millennium (1996-99), dans l'épisode éponyme (7. 4) 
eu de cesse de repousser sa fin et ses limites, toutes les voies ne mènent nécessairement pas au succès...

\section{L'ordre du monde : le début et la fin comme sujets d'X- Files}

Comme nous le laissions entendre dans notre introduction, le début et la fin ne sont pas uniquement des problématiques touchant à la structure de la série et, a fortiori, à sa diffusion. Il faut tout de même avouer que ce dernier point offre une explication intéressante du mode de conclusion d'X-Files. Le succès aidant, la série n'a eu de cesse d'avancer et d'ouvrir de nouvelles voies devant sa mythologie, offrant intrigues et possibilités d'interprétations à ses téléspectateurs. En toute logique, son dénouement ne peut être que déceptif, la fin du monde annoncée lors du dernier épisode venant couper court à toute échappatoire, et ce même si la série se conclut sur la possibilité d'une bataille et sur une note d'espoir. Admettons toutefois que, comme bien souvent, cette fin n'en est pas totalement une et tendrait même à être dépassée. Le second film, plus clairement centré sur les personnages et leur évolution, ne permet pas de croire que les deux anciens agents livrent un combat, mais tentent plutôt de réchapper à leur passé. Au contraire même : ils sont reclus dans leur maison, et le changement de décor semble indiquer que les neuf années passées à collecter les preuves n'ont finalement été qu'illusions et quête spirituelle.

\subsection{Impossible de mourir}

$X$-Files n'a eu de cesse de repousser sa fin... La série cumule en effet les conclusions et les relances, au point de donner à croire que la série est clairement une "histoire sans fin». Le service des affaires non classées est régulièrement fermé pour finalement être rouvert, brûlé dans un incendie (The End, 5.20 ) avant de renaître de ses cendres (The Beginning, 6.1). Mulder cherche encore et toujours sa sœur, croyant parfois la retrouver pour finalement comprendre la supercherie dont il est à chaque fois la victime. Et lorsqu'il découvre enfin que Samantha est morte depuis de nombreuses années (Closure, 7.11), il semble que l'exercice du deuil va être un très long processus pour lui. Il en est de même pour le complot que les deux agents tentent de déjouer. Le Syndicat en charge de collaborer avec l'envahisseur extraterrestre est confondu puis vaincu au milieu de la sixième saison (One Son, 6.12). Mais de nouvelles révélations sur le sort de l'humanité débordent ensuite largement le cadre du complot, avant que celui-ci prenne une autre forme dans les deux dernières saisons. À la fin de la septième saison, lorsque le service des affaires non classées est audité afin de réduire ses dépenses et que constat est fait que les deux grandes enquêtes qui ont retenu les deux agents pendant plusieurs années 
semblent finalisées, Mulder affirme que beaucoup de questions restent en suspens et qu'ils doivent continuer leur travail. En somme, que rien n'est terminé. Cet épisode, intitulé Requiem (7.22) pour marquer le geste d'achèvement dont il est le porteur, est justement l'occasion d'un couple fin/relance de premier ordre. Alors qu'ils retournent sur les lieux de leur première enquête, à Bellefleur dans l'Oregon, Mulder et Scully constatent que l'entreprise qu'ils ont déjouée est toujours à l'œuvre. Surtout, la teneur même de l'accès aux preuves va changer, Mulder faisant alors l'expérience de l'enlèvement et touchant par-là la vérité qu'il cherche tant. La fin de la saison, qui aurait pu acter aussi la fin de la série, se mue donc en exploration de nouvelles voies de compréhension de soi et du monde. Avec en toile de fond le thème de la mort, la série joue bien souvent sur la problématique de la fin, faisant progressivement de ses deux personnages des héros au sens plein du terme. En effet, si Scully sort indemne de son enlèvement (One Breath, 2.8) et, surtout, si elle réchappe miraculeusement d'un cancer en phase terminale (Redux II, 5.2), Mulder, quant à lui, décède et revient d'entre les morts dans la huitième saison (Deadalive, 8.15, fig.4), avant de s'échapper de la prison où le verdict de sa mort par injection létale vient d'être prononcé (The Truth, 9.20). En clair, la série ne cesse de mettre en abyme son impossibilité à mourir.

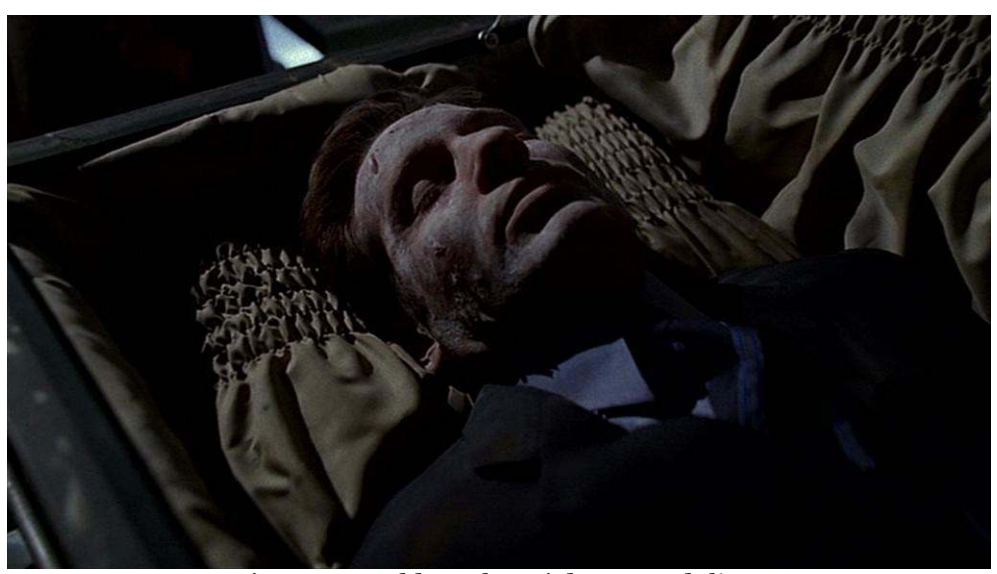

Figure 4 : Mulder exhumé dans Deadalive

\subsection{L'affaire non classée : la condition d'une pérennité}

À ce stade de la série, les créateurs tendent clairement à traiter deux échelles de la narration qui, chacune à leur manière, posent le problème du début et de la fin. D’une part, les deux agents ne se 
contentent plus de chercher des extraterrestres pour dévoiler aux yeux du monde leur existence : ils veulent expliquer les raisons de leur présence et se rendent progressivement compte qu'ils ont toujours été là. En somme, la série bouscule le problème du début et de la fin, pour poser ceux, plus métaphysiques, des origines et de la disparition, ce qui oblige les deux agents à pousser les barrières du temps pour trouver les preuves favorables à leur enquête. L'autre sujet central de la série est bien le lien singulier qui unit Mulder et Scully. Relation fusionnelle de confiance absolue, leur amour est resté platonique pendant de longues années afin de repousser sa consommation et de maintenir la tension existant entre eux. Même lorsque le spectateur apprend qu'ils ont eu un enfant ensemble, il n'en reste pas moins qu'ils ne s'autorisent pas à vivre leur amour de manière conventionnelle. Mulder s'enfuit pour poursuivre son enquête et devient un père absent face à Scully, la mère célibataire responsable et moderne, mais qui reste protectrice envers son amant de toujours (fig.5). Là est très certainement un des derniers facteurs du succès et de la pérennité d' $X$ Files dans le panorama des séries TV : apprendre des erreurs de ses prédécesseurs et, dans ce cas de figure, ne pas attraper le « syndrome » Clair de lune (1985-89).

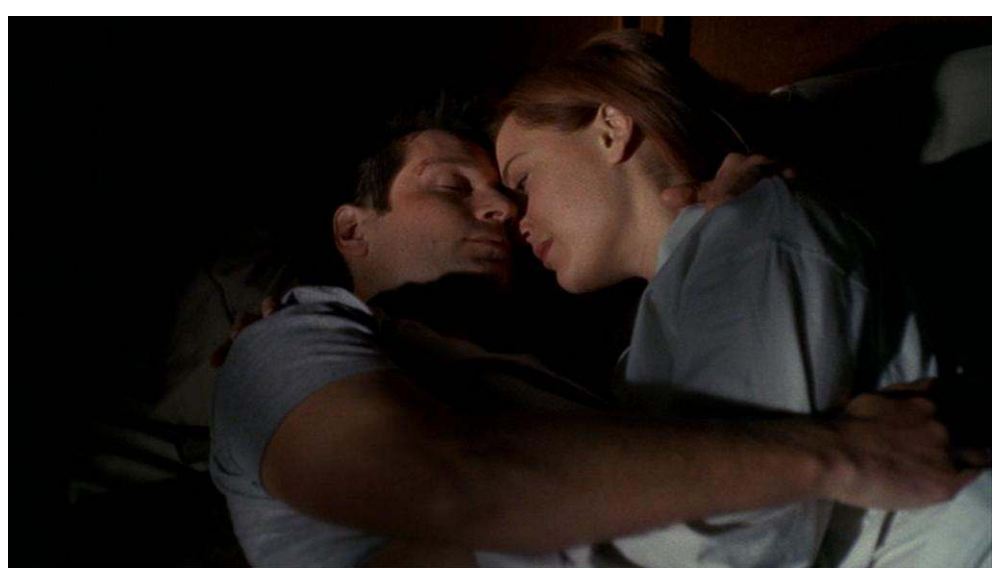

Figure 5 : Mulder et Scully formant enfin un couple dans The Truth (9.20)

Repousser sa fin n'est pas une mince affaire pour une série. Le cas d'X-Files montre toutes les difficultés de conclure, notamment face à un sujet qui ne cesse de s'étendre et de se complexifier. Les créateurs ont surtout fait preuve d'une inventivité rare pour maintenir une tension narrative forte et entretenir la mythologie. C'est, quelque part, l'idée de deux séries en une qui a permis de ménager et de ne pas trop 
lasser le téléspectateur : un feuilleton fortement marqué par un sujet, les extraterrestres, et irrigué par la veine des théories du complot ; une série, qui frise parfois l'anthologie, se voulant multiples variations sur les genres cinématographiques. Elle a aussi réussi le tour de force du recul critique, en jouant sur ses propres codes avec ironie et un humour pince-sans-rire redoutable pour se relancer sans cesse, mêler dénouement et ouverture en brouillant ses seuils narratifs et diégétiques, mais aussi ses codes esthétiques. En somme, faire d'ellemême un dossier jamais totalement fermé, une véritable affaire non classée, que la dixième saison ne manquera pas de rouvrir.

\section{Bibliographie}

GAI Frédéric, «The $X$-Files : allégorie de la condition postmoderne du monde », in TV/Series, $\mathrm{n}^{\circ} 1$, http://www.univ-lehavre. fr/ulh_services/Numero-1-Issue1.html.

RUFFEL Lionel, Le Dénouement, Lagrasse, coll. « Chaoïd », Verdier, 2005.

Frédéric Gai est docteur en lettres modernes de l'Université de Caen. Travaillant dans l'édition, il poursuit ses recherches sur l'œuvre de François Mauriac, mais aussi sur la littérature sportive. Il rédige actuellement un ouvrage sur The X-Files.

Frédéric Gai has a Ph.D in modern literature from the University of Caen. Working in publishing, he continues his research on François Mauriac, and on sports literature. He is currently writing a book on The X-Files. 